\title{
BCR/ABL1 Fusion Protein with ABL1 NP_005148.2:p.T315A
}

National Cancer Institute

\section{Source}

National Cancer Institute. BCR/ABL1 Fusion Protein with ABL1 NP 005148.2:p.T315A. NCI Thesaurus. Code C153115.

A fusion protein encoded by the BCR/ABL1 fusion gene with a mutation in codon 315 of the ABL1 gene. This protein is comprised of the N-terminus of the breakpoint cluster region protein fused to almost the entire tyrosine-protein kinase ABL1 protein where the threonine at position 315 of the ABL1 protein has been replaced by alanine. 